\title{
SECTOR DE HIERBAS AROMÁTICAS Y CERTIFICACIÓN DEL SISTEMA DE GESTIÓN DE INOCUIDAD ALIMENTARIA ISO 22000
}

\author{
M. Almeida ${ }^{1}$, Universidad Tecnológica Equinoccial
}

Recepción/Received: 2011-03-09

Aceptación/Accepted: 2011-07-11

Publicado/Published: 2011-08-05

Resumen -El presente trabajo tiene como objetivo analizar el por qué una buena parte de las empresas pertenecientes al sector de hierbas aromáticas, no han podido concretar la implementación de su sistema de gestión de inocuidad alimentaria ISO 22000 y conseguir su certificación. Para posibilitar una mejor comprensión, este artículo fue organizado de la siguiente manera: inicialmente, se realiza una descripción del fundamento teórico respecto a la cadena productiva y agroalimentaria, el marco normativo y el comercio exterior, luego se describe la metodología de investigación utilizada, más adelante se presenta una reseña de los informes de las empresas consultoras que participaron en la fases uno y dos de los proyectos cofinanciados por la CAF-MICIP-CORPEI, cuyo objetivo fundamental fue el de fortalecer la competitividad de la cadena de plantas medicinales, aromáticas y derivados, para afianzar su participación en los mercados de Estados Unidos de Norteamérica, Canadá y América Latina; en los cuales han participado las empresas/organizaciones motivo de este estudio (SUMAK LIFE-ERPE, JAMBI KIWA, CETCA, UNORCACHT, AROMAS DEL TUNGURAHUA y FUNDACIÓN CHANKUAP) y finalmente se describe el logro alcanzado por las empresas objeto de estudio respecto a los requisitos de las normas, así como los resultados de una entrevista realizada a informantes calificados de empresas/ organizaciones respecto al estado actual de sus sistemas, con base a esta información se emiten las conclusiones y recomendaciones.

Palabras clave -Buenas Prácticas Agrícolas (GlobalGAP), Buenas Prácticas de Manufactura (BPM), Análisis de Peligros y Puntos Críticos de Control (HACCP/ APPCC), Sistema de Gestión de Inocuidad Alimentaria (ISO 22000), Biocomercio.

Abstract -The present investigation's objective is to analyze why most companies that work with aromatic herbs have not been able to establish their ISO 22000 nutrition harmlessness management system and get the corresponding certifications.

To understand much better the content, this article was organized as follows: First, there is a full description of the theoretical frame referring to the productive, agribusiness and food chain, regulations frame and international trade There is a description of the research methodology which was used. There is a review of the reports set forth by the advisor companies which participated on stages 1 and 2 of projects jointly financed by CAF - MICIP - CORPEl; whose main objective was to empower competitiveness of medicinal plants chain, aromatic herbs and derivatives, to ensure their participation in international markets such as the USA, Canada, and Latin America, in which the companies and organizations mentioned on this work have already participated, for instance SUMAK LIFE - ERPE, JAMBI, KIWA, CETCA, UNORCACHT, AROMAS DEL TUNGURAHUA AND FUNDACION CHANKUAP.

Finally, there is a description of the achievement gotten by the companies mentioned on this article referring to regulation requirements, as well as the results of an interview made to some qualified informants coming from the companies, such interview was about the current condition of their systems, conclusions and recommendations are submitted on the basis of this information.

Keywords -Balance of payments, Cuba, Current acount, depression, economy, Granma, stockbreding, USSR, Zafra.Nectati aped ut illorit adipid enes ame nus. 


\section{INTRODUCCIÓN}

El sector de plantas medicinales, aromáticas y derivados en el Ecuador viene presentando un crecimiento sostenido con muchas posibilidades de desarrollar productos para la exportación, siendo sus principales mercados el alimenticio, el farmacéutico y el cosmético.

La producción de hierbas aromáticas y medicinales, se encuentra presente en diversas regiones del territorio nacional, esta dispersión hace que cada especie cuente con las características propias de la zona en la que es cultivada, motivado ello por las condiciones edáficas, climáticas y culturales propias de cada región.

Actualmente el cultivo de hierbas aromáticas y medicinales en gran parte es comunitaria y se ha convertido en un importante componente del ingreso de pequeños y medianos productores rurales, especialmente de mujeres con conocimientos ancestrales sobre plantas aromáticas y medicinales.

Respecto al comercio mundial de hierbas aromáticas, se puede decir que se encuentra en un crecimiento sostenido y moderado que responde, entre otros factores, a la tasa de crecimiento de la población; al mayor consumo de infusiones aromáticas; al aumento en la demanda de alimentos naturales exentos de saborizantes, aditivos y conservantes sintéticos entre otros ${ }^{2}$.

Según la iniciativa Biocomercio sostenible, las hierbas aromáticas (plantas medicinales) pueden ser parte del biocomercio al producirse de manera orgánica con demanda en comercio justo y ecológico, siendo las principales restricciones de ingreso a los países desarrollados las normas de empaque, etiquetado y eco-etiquetado, normas de calidad y ambientales a parte de las normas propias de cada país ${ }^{3}$.

De acuerdo a la información de CORPEl, "Ecuador dispone de aproximadamente 500 especies de plantas medicinales, de las cuales 228 son las más utilizadas y 125 son las más comercializadas. Se estima que la superficie cultivada con plantas aromáticas y medicinales son aproximadamente 600 hectáreas..."

2 Subsecretaría de Comercio Internacional. (2008). Informe sector alimentos industrializados. Infusiones y Especias. Buenos Aires. Argentina

3 Programa de Apoyo a la Gestión de Comercio Exterior. BIP-MICIP (2007) Propuesta para negociaciones comerciales sobre bienes ambientales ante la OMC. Marcia Almeida. Quito. Ecuador.
En este contexto, a partir del año 2006, empresas del sector de plantas medicinales, aromáticas y derivados han venido atravesando procesos de capacitación e implementación de sistemas de control de calidad y mejoramiento de la calidad a través de proyectos cofinanciados por la CAF-MICIP-CORPEI, cuyo objetivo fundamental fue fortalecer la competitividad de esta cadena productiva para afianzar su participación en los mercados de Estados Unidos de Norteamérica, Canadá y América Latina.

Entre las empresas que participaron en las dos fases de estos proyectos fueron SUMAK LIFE - ERPE, JAMBI KIWA, CETCA, ILE, UNORCACHT, AROMAS DEL TUNGURAHUA y FUNDACIÓN CHANKUAP, cabe mencionar que las tres últimas participaron únicamente en la segunda fase del proyecto e ILE únicamente en la primera fase, también es necesario señalar que a excepción de CETCA y Aromas del Tungurahua el resto de organizaciones representan proyectos de desarrollo comunitario que promueven e incentivan el cultivo de hierbas aromáticas.

Estos proyectos desarrollados en dos fases han tenido impacto sobre las actividades referentes a poscosecha en campo y poscosecha en la planta de procesamiento así como en las actividades de procesamientos de plantas medicinales, aromáticas y derivados, en las que se ha buscado el mejoramiento de la gestión de calidad en base al cumplimiento de los requisitos de las normas de GlobalGAP (Versión 3.1 de septiembre del 2007, conocida anteriormente como EUREPGAP, para campo), BPM (Buenas Prácticas de Manufactura), HACCP/APPCC (Análisis de Peligros y Puntos Críticos de Control) e ISO 22000:2005 (Sistema de Gestión de Inocuidad Alimentaria).

Sin embargo, a pesar de que las dos fases de los proyectos se llevaron a cabo exitosamente, las empresas participantes aún no han logrado culminar la implementación de los sistemas y obtener sus certificaciones ISO 22000 y GlobalGAP.

Con estos antecedentes, el objetivo principal de esta investigación es responder, el por qué una buena parte de las empresas pertenecientes a este sector no han logrado concretar aún de manera efectiva la implementación y certificación de su sistema de gestión de inocuidad alimentaria ISO 22000, que involucra tanto a las Buenas Prácticas de Manufactura (dentro de su programa de prerrequisitos) como a los principios del HACCP, si de antemano se conoce que la calidad e inocuidad de sus productos es un requisito fundamental para competir exitosamente en el mercado global. 


\section{FUNDAMENTOS TEÓRICOS}

Se describen los principales conceptos involucrados en esta investigación, tales como la cadena productiva, la cadena agroalimentaria, marco normativo y comercio exterior.

\section{La cadena productiva}

Según CORPEl, "el sector está conformado por productores, procesadores y comercializadores de materia prima, de productos semi-elaborados y elaborados. En el área de producción se cuentan con 2300 productores primarios asociados y 3 productores privados. En el área de transformación existen 8 organizaciones de pequeños productores y 10 PYMES industriales...".

El sector primario se compone de pequeños y microproductores que poseen cultivos de una hectárea o menos, con lo cual, luego es necesaria la actividad de intermediarios quienes, al contactar a unos cuantos de esos productores, actúan como acopiadores como es el caso de SUMAK LIFE, JAMBI KIWA, FUNDACIÓN CHANKUAP, llegando de esta manera a los mercados nacionales e internacionales con volúmenes más o menos importantes, satisfaciendo la eventual demanda.

\section{La cadena agroalimentaria}

Desde el punto de vista de la realidad socioeconómica "la cadena agroalimentaria es un sistema que agrupa actores económicos y sociales interrelacionados que participan articuladamente en actividades que agregan valor a un bien o servicio, desde su producción hasta que este llega a los consumidores, incluidos los proveedores de insumos y servicios, transformación industrialización, transporte, logística y otros servicios de apoyo, como el de financiamiento".

En el caso del sector de hierbas aromáticas, comprende las diferentes fases en la producción (preparación del suelo, protección del cultivo, cosecha, transporte, poscosecha en campo, poscosecha en la planta de procesamiento) hasta obtención de la materia prima, el procesamiento de la misma, obteniendo el producto terminado industrializado omo son las infusiones aromáticas y te aptos para el consumo humano, mismos que son distribuidos y comercializados hasta llegar a los consumidores finales.

Las cadenas agroalimentarias también han sido utilizadas para lograr la incorporación de actores o eslabones débiles a los mercados o para que productores pequeños puedan abastecer mercados en condiciones más favorables.

La ISO 22000:2005 define a la cadena alimentaria como: "Secuencia de las etapas y operaciones involucradas en la producción, procesamiento, distribución, almacenamiento y manipulación de un alimento y sus ingredientes, desde su producción primaria hasta el consumo".

\section{MARCO NORMATIVO}

Según el Decreto Ejecutivo No.3253 publicado en el Registro Oficial 696 vigente desde Noviembre del 2004: las Buenas Prácticas de Manufactura (BPM), son de cumplimiento obligatorio en nuestro país, y constituyen "principios básicos y prácticas generales de higiene en la manipulación, preparación, elaboración, envasado y almacenamiento de alimentos para consumo humano, con el objeto de garantizar que los productos se fabriquen en condiciones sanitarias adecuadas y se disminuyan los riesgos inherentes".

En cambio GlobalGAP (Versión 3.1 de septiembre del 2007, conocida anteriormente como EUREPGAP, para campo), el Sistema de Análisis de Peligros y Puntos Críticos de Control HACCP/APPCC, así como la ISO 22000:2005 son normas internacionales, de carácter voluntario, sin embargo el cumplimiento de sus requisitos es requerido para exportar a EEUU, Canadá y la Unión Europea, entre otros destinos.

Analizando estas normas internacionales, vemos que el HACCP "es una aproximación sistemática para la prevención de los riesgos (biológicos, químicos y físicos) asociados a la seguridad de los alimentos". El Sistema de Gestión de Inocuidad de Alimentos SGIA ISO 22000, "es un sistema de gestión para dirigir y controlar una organización en lo relativo a la inocuidad alimentaria" y GlobalGAP, "es un programa y una referencia global para las Buenas Prácticas Agrícolas (BPA).

Básicamente el sistema de gestión de inocuidad alimentaria conlleva actitudes preventivas por parte de quienes participan de la cadena agroalimentaria, por lo que conlleva a la obtención de productos alimenticios inocuos (según la ISO 22000:2005, "la inocuidad de los alimentos es un concepto que implica que un alimento no causará daño al consumidor cuando se prepara y/o consume de acuerdo con su uso previsto) y competitivos 
en un marco de mejora continua a través de la adopción de mecanismos de autocontrol por parte de la empresa/ organización".

Es de esperar que los sistemas de control y mejoramiento de calidad, así como el sistema de gestión de inocuidad alimentaria permitan desarrollar un modelo en donde la inocuidad, la calidad, la productividad y la mejora continua se integran para generar una mayor confianza de los consumidores y el incremento de los beneficios para las empresas, propiciando un clima de mejoramiento de las relaciones cada vez más eficientes entre los intervinientes en la cadena agroalimentaria.

\section{Comercio exterior}

El Informe Mercado de los Estados Unidos de América: Oportunidades para Ecuador en Infusiones Aromáticas Medicinales y Cosméticos Naturales para el Cuidado de la Piel de Santiago García Álvarez (octubre 2008) sostiene lo siguiente:

Ecuador requiere internacionalizar con mayor fuerza varios productos que surgen de su situación privilegiada de disponibilidad de recursos naturales, si bien el país ocupa el séptimo puesto en mega diversidad en el mundo, aquello no es suficiente en el sentido de que, lo importante, es encontrar los medios necesarios para que esa riqueza natural sea aprovechada de manera sostenible a nivel ambiental y económico.

Precisamente, uno de los sectores que ha tomado cuerpo y que presenta una situación expectante de crecimiento lo constituye el de productos e ingredientes naturales, conformado por la producción de hierbas aromáticas y medicinales, infusiones, té, colorantes, saborizantes y aceites esenciales.

La participación del mencionado sector en el mercado nacional mantiene una evolución creciente en el tiempo, también muchos han sido los avances en materia de exportaciones, gracias al propio esfuerzo de las empresas y organizaciones comunitarias involucradas en este importante segmento productivo, como también a los esfuerzos institucionales privados, públicos y de la cooperación internacional.

\section{METODOLOGÍA DE LA INVESTIGACIÓN}

Se realizó una investigación de tipo cualitativo, siendo una de las estrategias utilizadas para obtener información la revisión de los informes de las empresas consultoras participantes en las dos fases de implementación del proyecto. Otra estrategia fue la realización de entrevistas a informantes calificados, tales como representantes de las empresas/organizaciones consideradas en el estudio tales como CETCA (Compañía Ecuatoriana del Té), ubicada en provincia de Pichincha; Fundación Chankuap, ubicada en la provincia de Morona Santiago; Sumak Life y Jambi Kiwa, ubicadas en la provincia de Chimborazo y Aromas del Tungurahua, ubicada en la provincia de Tungurahua; se entrevistó además a representantes del organismo ejecutor como la CORPEl y Corporación 3D Calidad como la empresa consultora involucrada en la asesoría y capacitación del sector.

Para la investigación se siguió un código de ética basada en la autenticidad de la información recolectada y respetando la confidencialidad de la misma.

El equipo investigador estuvo conformado únicamente por la autora de este artículo.

RESULTADOS: ANÁLISIS DE LOS INFORMES DE LAS EMPRESAS CONSULTORAS

\section{Análisis del informe de consultoría de la fase uno}

Según el informe emitido por CHEM Consultores, empresa consultora que participó en la primera fase del proyecto "Fortalecer la competitividad de la cadena de plantas medicinales, aromáticas y derivados y afianzar su participación en los mercados de Estados Unidos de Norteamérica, Canadá y América Latina". Componente 3: "Implementación de Sistemas de Control de Calidad y Mejoramiento de la Calidad de Poscosecha", Los objetivos generales estuvieron enfocados en primer lugar a mejorar el sistema de aseguramiento de calidad sanitaria en las empresas participantes, garantizando la calidad de las materias primas y materiales, el procesamiento controlado a través de personal capacitado, infraestructura adecuada, maquinaria y equipos apropiados y calibrados, métodos estandarizados y controlados que aseguren la elaboración de un producto final inocuo y apto para el consumo humano.

En segundo lugar se buscó transmitir a los participantes el conocimiento y los elementos necesarios para la implementación y mantenimiento de un sistema eficaz de aseguramiento de calidad sanitaria.

Analizando los resultados del informe se evidencia que el proyecto llega a cumplir los objetivos generales respecto a la capacitación y levantamiento de documentación, quedando como tarea a las empresas el continuar con la implementación.

De manera general en esta primera fase el nivel de cumplimento de las empresas/organizaciones motivo de este estudio respecto a los requisitos de los sistemas BPM y HACCP se describen en la siguiente tabla: 
TABLA No.1

RESULTADOS DEL PROYECTO PRIMERA FASE

\begin{tabular}{|l|l|l|}
$\begin{array}{c}\text { EMPRESA/ } \\
\text { ORGANIZACIÓN }\end{array}$ & \multicolumn{1}{|c|}{$\begin{array}{c}\text { CUMPLIMIENTO } \\
\text { REQUISITOS BPM }\end{array}$} & \multicolumn{1}{c|}{$\begin{array}{c}\text { CUMPLIMIENTO } \\
\text { REQUISITOS HACCP }\end{array}$} \\
\hline FUNDACIÓN/ CHANKUAP & No participó en esta 1era. fase & No participó en esta 1era. fase \\
\hline CETCA & $88.5 \%$ & $\begin{array}{l}\text { No partició en la implementación } \\
\text { de este sistema }\end{array}$ \\
\hline JAMBI KIWA & $74.9 \%$ & $\begin{array}{l}\text { No participó en la implementación } \\
\text { de este sistema }\end{array}$ \\
\hline UNORCACHT & No participó en esta 1era. fase & No participó en esta 1era. fase \\
\hline $\begin{array}{l}\text { AROMAS DEL } \\
\text { TUNGURAHUA }\end{array}$ & No participó en esta 1era. fase & No participó en esta 1era. fase \\
\hline ILE & $62.5 \%$ & $86.7 \%$ \\
\hline SUMAK LIFE & $76.3 \%$ & $50 \%$ \\
\hline
\end{tabular}

Fuente: CORPEI. Informe final de actividades de CHEM Consultores. Implementación de Sistemas de Control de Calidad en el Sector de Ingredientes y Productos Naturales. Fase 1. Quito, Ecuador. Trabajo sin publicación.

\section{La empresa consultora declara en su informe que:}

"tras los 10 meses de asesoría, las empresas participantes en el proyecto, han conseguido superar en mayor o menor porcentaje, las barreras técnicas y de bajo cumplimiento, para alcanzar niveles importantes en el aseguramiento de la sanidad e inocuidad de los productos alimenticios que procesan y que en todas las empresas los resultados han sido satisfactorios, ya que no solamente se ha logrado la implantación de una cultura de calidad sanitaria e inocuidad, sino que también se ha alcanzado mejor sistematización y organización de los procesos". Entre las recomendaciones emitidas se menciona continuar con la implementación y mantenimiento de BPM y HACCP, así como el fomentar la participación asociativa de las empresas/organizaciones del sector respecto a la estandarización de especificaciones, implementación de laboratorios de control de calidad especializados, intercambiar auditores internos, controlar y optimizar los procesos.

\section{Análisis del informe de consultoría de la fase dos}

En el mes de marzo del año 2008, la empresa consultora C3D Calidad, continuó con la fase dos del "Proyecto de Implementación de Sistemas de Control de Calidad en el Sector de Ingredientes y Productos Naturales", en esta fase se contó con la participación de seis empresas representantes del sector entre ellas, JAMBI KIWA, SUMAK LIFE, AROMAS DEL TUNGURAHUA, CETCA, FUNDACIÓN CHANKUAP Y UNORCACHT.

Entre los objetivos generales que se perseguía en esta segunda fase estaban el de apoyar la mejora de sistemas de control de calidad utilizados por las empresas del sector de Ingredientes y Productos Naturales y el de desarrollar en las empresas beneficiarias del proyecto CAF-MIC-
CORPEl un sistema documental que permitan la difusión y sistematización de estándares y normas de calidad para el sector de Ingredientes y Productos Naturales.

Entre los objetivos específicos se buscaba implementar un Sistema de Gestión Integrado de Calidad de las normas ISO 22000 (Sistema de Gestión de Inocuidad Alimentaria) y Buenas Prácticas agrícolas (GlobalGAP).

Los resultados alcanzados en la implementación del SGIA ISO 22000:2005 se presentan en la siguiente tabla:

TABLA No. 2

RESULTADOS DEL PROYECTO SEGUNDA FASE

\begin{tabular}{|l|c|c|c|}
$\begin{array}{c}\text { EMPRESA/ } \\
\text { ORGANIZACIÓN }\end{array}$ & $\begin{array}{c}\text { CUMPLIMIENTO } \\
\text { REQUISITOS BPM }\end{array}$ & $\begin{array}{c}\text { CUMPLIMIENTO } \\
\text { REQUISITOS HACCP }\end{array}$ & $\begin{array}{c}\text { CUMPLIMIENTO REQUISITOS } \\
\text { GLOBALGAP (DEBES MENORES) }\end{array}$ \\
\hline FUNDACIÓN/ CHANKUAP & $66.2 \%$ & No aplica & No aplica \\
\hline CETCA & $62.1 \%$ & No aplica & No aplica \\
\hline JAMBI KIWA & $78.3 \%$ & $95 \%$ & $85 \%$ \\
\hline UNORCACHT & $64.8 \%$ & $92 \%$ & $62 \%$ \\
\hline $\begin{array}{l}\text { AROMAS DEL } \\
\text { TUNGURAHUA }\end{array}$ & $65.7 \%$ & $63 \%$ & $38 \%$ \\
\hline SUMAK LFE & $75.9 \%$ & $100 \%$ & $96 \%$ \\
\hline
\end{tabular}

Fuente: CORPEI. Informe final de actividades de Corporación3D Calidad. Implementación de Sistemas de Control de Calidad en el Sector de Ingredientes y Productos Naturales. Fase 2. Quito, Ecuador. Trabajo sin publicación.

Corporación 3D Calidad (2009) declara como resultados los siguientes:

"Se desarrollaron y documentaron Estándares de Calidad de materia prima usada para los productos más comercializados por las empresas entre ellos podemos mencionar manzanilla, menta, toronjil y tomillo, los cuales fueron revisados y aprobados por las empresas participantes y CORPEl".

Los eventos de capacitación sobre los requisitos de las normativas y la importancia y la pertinencia de la aplicación de los sistemas para el sector alimenticio, se realizaron en las instalaciones de CORPEI, se formaron y calificaron auditores internos para los sistemas ISO 22000:2005 y Buenas Prácticas Agrícolas y se ejecutaron dos auditorías internas lo que permitió medir el avance de la implementación de los sistemas en sus diferentes fases, realizándose además un evento de intercambio de experiencias de resultados obtenidos en la primera auditoría interna.

Se entregó un informe final que contiene toda la información de las actividades realizadas con sus respectivos respaldos.

Entre las recomendaciones emitidas por la empresa consultora se menciona:

"Gestionar fondos con organismos de cooperación y gubernamentales para ayudar a que las empresas participantes mejoren su infraestructura para dar cumplimiento cabal a las Buenas Prácticas de Manufactura y GlobalGAP". 
"Buscar fondos con organismos de cooperación y gubernamentales para llevar a Las empresas participantes a certificar ISO 22000 y GlobalGAP".

"Ante la dificultad por parte de los líderes del equipo de inocuidad y alta dirección para trabajar en los sistemas debido a que deben atender muchas actividades del trabajo diario, y no queda tiempo para documentar e implementar los sistemas. Se recomienda trabajar con eficacia (satisfaciendo a los clientes) y con eficiencia (haciendo las cosas bien y utilizando adecuadamente todos los recursos), pues esta es la manera natural y lógica de hacer las cosas en cualquier actividad de la organización. Sin embargo muchas organizaciones le llaman trabajo diario a apagar incendios, solucionar problemas inmediatos, manejar crisis; y al estar ocupados en ello, lógicamente no tendrán tiempo para nada más; pero es porque no hacen las cosas bien desde la primera vez".

"En base a los resultados positivos obtenidos, se recomienda continuar con el programa de calidad puntualmente con componentes como apoyo técnico en el layout (diseño y rediseño de las plantas de alimentos), Asistencia técnica para terminar de llevar a las empresas al cumplimiento de todos los requisitos de ISO 22000 y GlobalGAP para que queden listas para obtener las certificaciones".

\section{Análisis de la información actual de las empresas}

Se consigue información directa de algunas empresas/organizaciones que participaron en el "Proyecto de Implementación de Sistemas de Control de Calidad en el Sector de Ingredientes y Productos Naturales", a través de una entrevista a CETCA, SUMAK LIFE ERPE, JAMBI KIWA, FUNDACIÓN CHANKUAP Y AROMAS DEL TUNGURAHUA, Para lo cual se realizaron las siguientes actividades: se elaboró un formato de entrevista, se contacto telefónicamente a cada una de las empresas participantes, se envió por correo electrónico el formato de la entrevista para que lo llenen.

Al recopilar la información se obtienen los hallazgos descritos en la siguiente tabla:

TABLA No. 3

\begin{tabular}{|l|l|c|l|}
\multicolumn{1}{|c|}{$\begin{array}{c}\text { EMPRESA/ } \\
\text { ORGANIZACIÓN }\end{array}$} & $\begin{array}{c}\text { PERSONA } \\
\text { ENTREVISTADA }\end{array}$ & $\begin{array}{c}\text { CUMPLIMIENTO DE } \\
\text { REQUISITOS DEL SGI } \\
\text { ISO 22000 } \\
\text { (SEGUN LA UTIMA } \\
\text { AUDITORIA INTERNA) }\end{array}$ & $\begin{array}{c}\text { CERTIFICACIÓNES } \\
\text { DISTINTAS A ISO 22000 } \\
\text { CON LAS QUE CUENTAN }\end{array}$ \\
\hline FUNDACIÓN/CHANKUAP & Jefe Control de Calidad & $54 \%$ & Biocomercio Rain Forest \\
\hline CETCA & Jefe de Planta & $62.1 \%$ & HACCP \\
\hline JAMBI KIWA & Gerente & $75,9 \%$ & Fair Trade BCS \\
\hline UNORCACHT & No se consigue la entrevista & $64.8 \%$ & No reporta \\
\hline $\begin{array}{l}\text { AROMAS DEL } \\
\text { TUNGURAHUA }\end{array}$ & Jefe de planta & $65.7 \%$ & No reporta \\
\hline SUMAK LIFE & $\begin{array}{l}\text { Coordinador de Calidad } \\
\text { yProducción }\end{array}$ & $82 \%$ & BCS \\
\hline
\end{tabular}

Fuente: Entrevistas a organizaciones que han participado de manera activa en el "Proyecto de Implementación de Sistemas de Control de Calidad en el Sector de Ingredientes y Productos Naturales", ejecutado por la CORPEI, y financiado por la CAF, MIC y CORPEl.

Las empresas ${ }^{4}$ manifiestan que sus sistemas ISO 22000 Y GlobalGAP se han mantenido respecto al sistema documental pero no se ha avanzado significativamente en la implementación y mejora continua, en su mayoría no han continuado con su programa de auditorías internas.

Entre las principales razones que mencionan las empresas y por las que aún no han logrado las certificaciones son principalmente por la falta de recursos económicos para realizar mejoras en su infraestructura, equipos y materiales de trabajo, la falta de continuidad en la implementación, dificultades logísticas, así como el hecho de que las personas que se capacitaron para implementar el sistema tienen múltiples funciones y no disponen de mucho tiempo para el sistema, otras se han retirado de las instituciones y las que han quedado al frente conocen muy poco de los sistemas.

Las empresas investigadas coinciden en su deseo de obtener las certificaciones pues creen que es una carta de presentación muy importante así como una garantía de calidad de sus productos, consideran además el costo de las certificaciones como poco accesible para las empresas pequeñas.

Entre las propuestas que plantean para que se les ayude están principalmente el conseguir financiamiento con planes de pago directo o a través de su asociación conseguir una especie de paquete que minimice los costos y todas las empresas puedan ser beneficiadas, asesorías individualizadas para cada empresa participante que busquen pulir las deficiencias, mantener el sistema y mejorarlo para acceder a las certificaciones, crear concienciación a nivel de la parte financiera de la necesidad de los sistemas, proponen además se consigan clientes que paguen la certificación.

\section{Análisis de la información obtenida de CORPEI}

En la entrevista realizada a la persona responsable de coordinar estos proyectos en CORPEl que fue el organismo ejecutor, manifiesta que se desarrolló otro proyecto para una tercera fase en la cual como uno

4 Empresas que han participado de manera activa en el "Proyecto de Implementación de Sistemas de Control de Calidad en el Sector de Ingredientes y Productos Naturales", ejecutado por la CORPEI, y financiado por la CAF, MIC y CORPEI 
de los objetivos generales era lograr que las empresas participantes certificaran su sistema de inocuidad alimentaria, este proyecto se presentó y aprobó pero debido a decisiones políticas en las que CORPEl a lo mejor cierre sus operaciones no pudo firmar como ente ejecutor. Al comunicar esta situación a las empresas, estas decidieron buscar apoyo en la Cámara de Industriales de Pichincha, la cámara firmo y dio la garantía sin embargo exigía a las empresas afiliarse lo que no gusto a las empresas, debió a que estaban ya afiliadas a sus diferentes asociaciones y cámaras, lamentablemente el proyecto se perdió.

Respecto a la pregunta central de esta investigación, de por qué aún no logran certificar sus sistemas las empresas, el representante de CORPEI manifiesta que es por razones netamente económicas puesto que la infraestructura de las plantas de producción es inadecuada, no cumplen aún con el prerrequisito de ISO 22000 respecto a las buenas Prácticas de Manufactura y las empresas no disponen de dinero propio o financiado para trabajar en ello, a pesar que la en la segunda fase se realizaron estudios técnicos para la construcción de las plantas para FUNDACIÓN CHANKUAP Y UNORCHRAT

Otra razón es que en algunas de estas empresas/ organizaciones, los gerentes están supeditados a las decisiones de los Comités Directivos conformados por los pequeños productores que no tienen entre sus prioridades los sistemas de gestión por considerarlo como un costo y no como una inversión y no conocen a profundidad de que se trata y de las necesidades reales de la empresa. Otra razón es la alta rotación del personal que fue capacitado en las dos fases del proyecto.

\section{CONCLUSIONES Y RECOMENDACIONES}

La mayoría de empresas/organizaciones estudiadas, así como el organismo ejecutor coinciden en la respuesta de la pregunta central de por qué aún no logran certificar sus sistemas, la cual es que por razones económicas, ya que las empresas no disponen de dinero propio o financiado para invertir en la mejora de la infraestructura de sus plantas de procesamiento para dar cumplimiento al prerrequisito de ISO 22000 respecto a las Buenas Prácticas de Manufactura, que garantizan un ambiente higiénico a través de la cadena alimentaria respecto a la producción, manipulación y suministro de alimentos inocuos para el consumo humano.

Las empresas/organizaciones y el organismo ejecutor, mencionan además que la falta de continuidad para la consecución de la tercera fase del proyecto detuvo el proceso de implementación, esta falta de continuidad se debió en gran medida a que CORPEI no pudo firmar este proyecto (ya aprobado) como ente ejecutor debido a que no se conocía si continuaba o no en sus funciones.

Dentro de las empresas/organizaciones, no debe perderse de vista además la rotación del personal clave que fue capacitado, así como la necesidad del compromiso de la alta dirección y la participación del personal para continuar con la implementación y mantenimiento de los sistemas de gestión de inocuidad alimentaria ISO 22000 y GlobalGAP.

Solamente un esfuerzo conjunto y continuo por parte de los actores estratégicos del sector conllevará a alcanzar las certificaciones y los estándares de calidad exigidos por los clientes nacionales e internacionales.

Las empresas del sector de plantas medicinales aromáticas y derivados deben asegurar su permanencia en el mercado garantizando calidad sanitaria a sus consumidores es decir generando productos aptos para el consumo humano; asegurando la inocuidad de los productos (produciendo productos sanos que no enfermen), evitando a toda costa el riesgo de enfermedades o intoxicaciones, mejorando sus sistemas de aseguramiento de la calidad sanitaria, asegurando la calidad de su materia prima y cumpliendo con los requerimientos de calidad de mercados nacionales e internacionales.

Se recomienda buscar y conseguir financiamiento de fondos con organismos de cooperación y gubernamentales para la adecuación de su infraestructura, así como para la asistencia técnica que ayude a las empresas al cumplimiento de todos los requisitos para que queden listas para obtener las certificaciones en ISO 22000, misma que generara confianza en el cliente respecto a la inocuidad de los productos.

Se recomienda también conseguir efectivamente una participación asociativa del sector para suplir las necesidades de los clientes respecto a volúmenes de producción.

Finalmente, se recomienda tomar conciencia respecto a la responsabilidad social de las empresas con sus proveedores que vienen a ser los micro-productores. 


\section{BIBLIOGRAFIA}

[1] Codex Alimentarios. Recuperado el 26 de agosto del 2010 en: http://www.codexalimentarius.net/web/index_en.jsp

[2] CORPEI. Informe final de actividades de CHEM Consultores. Implementación de Sistemas de Control de Calidad en el Sector de Ingredientes y Productos Naturales. Fase 1. Quito, Ecuador. Trabajo sin publicación.

[3] CORPEl. Informe final de actividades de Corporación 3D Calidad. Implementación de Sistemas de Control de Calidad en el Sector de Ingredientes y Productos Naturales. Fase 2. Quito, Ecuador. Trabajo sin publicación.

[4] CORPEl. Informe Mercado de los Estados Unidos de América: Oportunidades para Ecuador en Infusiones Aromáticas Medicinales y Cosméticos Naturales para el Cuidado de la Piel. Quito, Ecuador. Trabajo sin publicación.

[5] El manual de publicación "APA" al alcance de todos. Recuperado el 7 de agosto del 2010 en: http://valencia.cc.fl. us/lrcwest/apapaper.html

[6] Hierbas aromáticas y medicinales y joyería: innovaciones para acceder a nichos especializados de mercado. Recuperado el 13 de septiembre del 2010 en:http://www. redproductiva.org/images/stories/innovaciones/j.KUENJOYAS239-265.pdf

[7] Subsecretaría de Comercio Internacional. (2008). Informe sector alimentos industrializados. Infusiones y Especias. Buenos Aires. Argentina.

[8] Programa de Apoyo a la Gestión de Comercio Exterior. BIP-MICIP (2007) Propuesta para negociaciones comerciales sobre bienes ambientales ante la OMC. Quito. Ecuador.

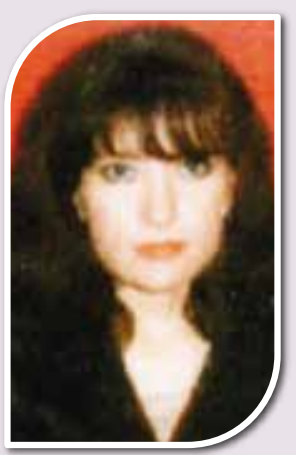

Marcia Almeida Guzmán

Bioquímica Farmacéutica de la Universidad Central del Ecuador 1992; Doctora en Bioquímica y Farmacia de la Universidad Central del Ecuador 1997; Especialista Superior en Mercadeo de la Universidad Andina Simón Bolívar, sede Ecuador 2001; Máster en Dirección de Empresas de la Universidad Andina Simón Bolívar, sede Ecuador 2003; Máster en Sistemas de Gestión Integrados: Calidad, Medio Ambiente y Riesgos Laborales en la EOI, Escuela de Negocios. Madrid-España 2006.

Profesora en programas de posgrado de la Universidad Tecnológica Equinoccial. 